\title{
EchoGéo
}

27 | 2014

Structures et armatures urbaines

\section{Environnement et géomatique : une palette variée de métiers, présentés à travers cinq entretiens}

Cécile Huet, Nicolas Payet et Lydie Goeldner-Gianella

\section{OpenEdition}

\section{Journals}

Édition électronique

URL : https://journals.openedition.org/echogeo/13785

DOI : 10.4000/echogeo.13785

ISSN : 1963-1197

Éditeur

Pôle de recherche pour l'organisation et la diffusion de l'information géographique (CNRS UMR 8586)

Référence électronique

Cécile Huet, Nicolas Payet et Lydie Goeldner-Gianella, «Environnement et géomatique : une palette variée de métiers, présentés à travers cinq entretiens », EchoGéo [En ligne], 27 | 2014, mis en ligne le 01 avril 2014, consulté le 10 août 2021. URL : http://journals.openedition.org/echogeo/13785 ; DOI : https://doi.org/10.4000/echogeo.13785

Ce document a été généré automatiquement le 10 août 2021.

EchoGéo est mis à disposition selon les termes de la licence Creative Commons Attribution - Pas d'Utilisation Commerciale - Pas de Modification 4.0 International (CC BY-NC-ND) 


\title{
Environnement et géomatique : une palette variée de métiers, présentés à travers cinq entretiens
}

\author{
Cécile Huet, Nicolas Payet et Lydie Goeldner-Gianella
}

1 Cinq professionnels de l'environnement utilisant régulièrement la géomatique ont accepté de nous parler de leur métier au cours d'entretiens réalisés en février 2014. Anciens étudiants de la licence professionnelle "Environnement et géomatique" ou du master 2 "Développement durable, management environnemental et géomatique", formés par l'Université Paris 1 et l'École Nationale des Sciences Géographiques, ils sont issus à l'origine de diverses disciplines et travaillent aujourd'hui dans des domaines variés du secteur de l'environnement, en utilisant régulièrement la géomatique. Deux d'entre eux, respectivement économiste et juriste, ont suivi le M2 DDMEG (Eddy Loo et Flora André) ; trois des interviewés ont suivi la licence professionnelle. L'une est géologue (Anne-Kyria Robin), deux autres sont géographes (Mathilde Le Duff et Kevin Lacan).

2 Le tableau suivant présente l'emploi qu'occupent actuellement ces cinq professionnels, en recherche ou en inspection/certification dans le domaine environnemental, dans les secteurs du transport ou des énergies renouvelables, ou dans une collectivité territoriale. 


\section{Eddy Loo (EL)}

Consultant en Hygiène, Sécurité, Environnement chez BUREAU VERITAS - leader mondial en Test/ Inspection/Certification. Présent dans plus de 140 pays, le Groupe rassemble plus de 50000 collaborateurs autour des domaines de la construction et de la gestion de patrimoine, de la certification, de la classification, du conseil, des inspections et audits, de la formation, des essais et analyses. Son métier de consultant l'amène au plus près de ses clients, puisqu'il les accompagne au cœur de leur activité pour ce qui relève de l'hygiène, de la sécurité ou de l'environnement. Les missions qu'il accomplit vont du conseil en système de management (qualité, sécurité, environnement, énergie, etc.) à l'audit de conformité réglementaire, en passant par l'évaluation de risques. De plus, il lui arrive d'intervenir sur des missions en Contrôle Technique de Construction, où il peut être amené, aux côtés de collaborateurs, à donner une expertise sur des ouvrages et bâtiments. Les missions couvertes dans ce domaine peuvent aller de l'inspection technique à l'assistance de maîtrise d'ouvrage.

\section{Anne-Kyria Robin (AKR)}

Doctorante au Laboratoire de Géographie Physique (LGP) de Meudon (UMR 8591 CNRS), Anne Kyria Robin conduit des recherches sur l'origine des obsidiennes - roches qui se forment en contexte volcanique et qui ont été utilisées durant la préhistoire pour la fabrication de lames de couteaux, haches, bijoux et miroirs. Cette thèse s'inscrit dans un questionnement archéologique financé par l'Agence Nationale de la Recherche: les archéologues s'interrogent sur l'origine des objets en obsidienne qui ont été diffusés de l'Égypte à la Géorgie, afin de reconstituer plus précisément les réseaux de diffusion de ces objets, après l'exploitation première des roches. Les travaux d'Anne Kyria Robin concernent plusieurs volcans d'Anatolie, à une échelle à la fois régionale et locale. Ses recherches s'opèrent dans un cadre interdisciplinaire : elle échange avec des archéologues, des chimistes, des géographes physiciens et des géologues. Après sa thèse, elle envisage de travailler dans l'enseignement et la recherche en poursuivant ses travaux dans le domaine volcanique, soit dans le champ des risques, soit dans celui de l'archéologie ou de la géomorphologie.

\section{Kevin Lacan (KL)}

Technicien d'étude SIG chez SYSTRA, leader mondial de l'ingénierie de transport public (principalement ferré) qui intervient à tous les niveaux de la chaîne de mobilité, des études amont d'implantation à l'exploitation et la maintenance des réseaux. Kevin Lacan intervient au département Développement des Outils de Production auquel est intégré le département Environnement de SYSTRA. Sa mission consiste à produire des cartes des nouveaux tracés ferroviaires intégrant les données environnementales, socio-économiques et topographiques fournies par les différents services de l'entreprise."

\section{Mathilde Le Duff (MLD)}

Chargée de mission chez Énergies et territoires développement (ETD), un bureau d'études indépendant travaillant au développement des énergies renouvelables qui compte un effectif de 8 ingénieurs et chargés de mission et dispose de 3 implantations en France (Landerneau, Roanne et Amiens). Les missions de Mathilde Le Duff sont diverses : elle réalise des supports cartographiques dans le cadre d'études d'implantation d'éoliennes, mais aussi des supports infographiques et de communication (poster, site internet...) et collecte, analyse et gère les données SIG de l'entreprise. 
Flora André (FA)

Chargée de mission au sein du pôle technique d'une communauté de communes de 30000 habitants, elle a été recrutée sur un poste contractuel à la suite de son stage de fin d'études. Ses principales missions consistent à mettre en œuvre deux politiques d'aménagement du territoire portées par la communauté de communes: une politique visant à améliorer la mobilité sur le territoire (transports en commun, liaisons cyclables, pôles d'échanges multi-modaux...) et une politique de gestion de l'affichage publicitaire permettant de protéger les paysages et les entrées de ville. La communauté de commune a en effet la particularité d'être un territoire littoral situé sur l'axe touristique qui relie la Baie du Mont St-Michel au Cap Fréhel.

\section{Des parcours de formations variés}

3 Bien que travaillant dans les métiers de l'environnement, ces professionnels ont eu des parcours de formation variés passant pour certains de l'environnement à la géomatique (AKR, KL), pour d'autres de la géomatique à l'environnement (MLD), ou ajoutant simplement cette compétence technique à leur formation initiale (EL et FA).

4 - AKR. Après une licence en géologie fondamentale dans la spécialité géodynamique et tectonique des plaques, je me suis intéressée aux risques, espérant trouver un emploi dans ce secteur. Ceci m'a conduit à suivre une licence professionnelle en "géomatique et environnement" pour progresser en géomatique et notamment en cartographie appliquée aux risques. J'ai ainsi effectué mon stage professionnel au Laboratoire de Géographie Physique (UMR 8591) sur le risque volcanique en Indonésie, cartographiant les dommages dus aux lahars ${ }^{1}$ du Mérapi sur l'île de Java. La carte qui suit (illustration 1), réalisée durant mon stage en L3 Pro, a été élaborée à partir d'une extraction d'une image satellite de données hydrographiques et de données sur le bâti, couplée à des observations de terrain géo-référencées et hiérarchisées, concernant l'extension de l'aléa et les dommages occasionnés. 
Illustration 1 - Dommages dus aux lahars du Mérapi sur l'île de Java

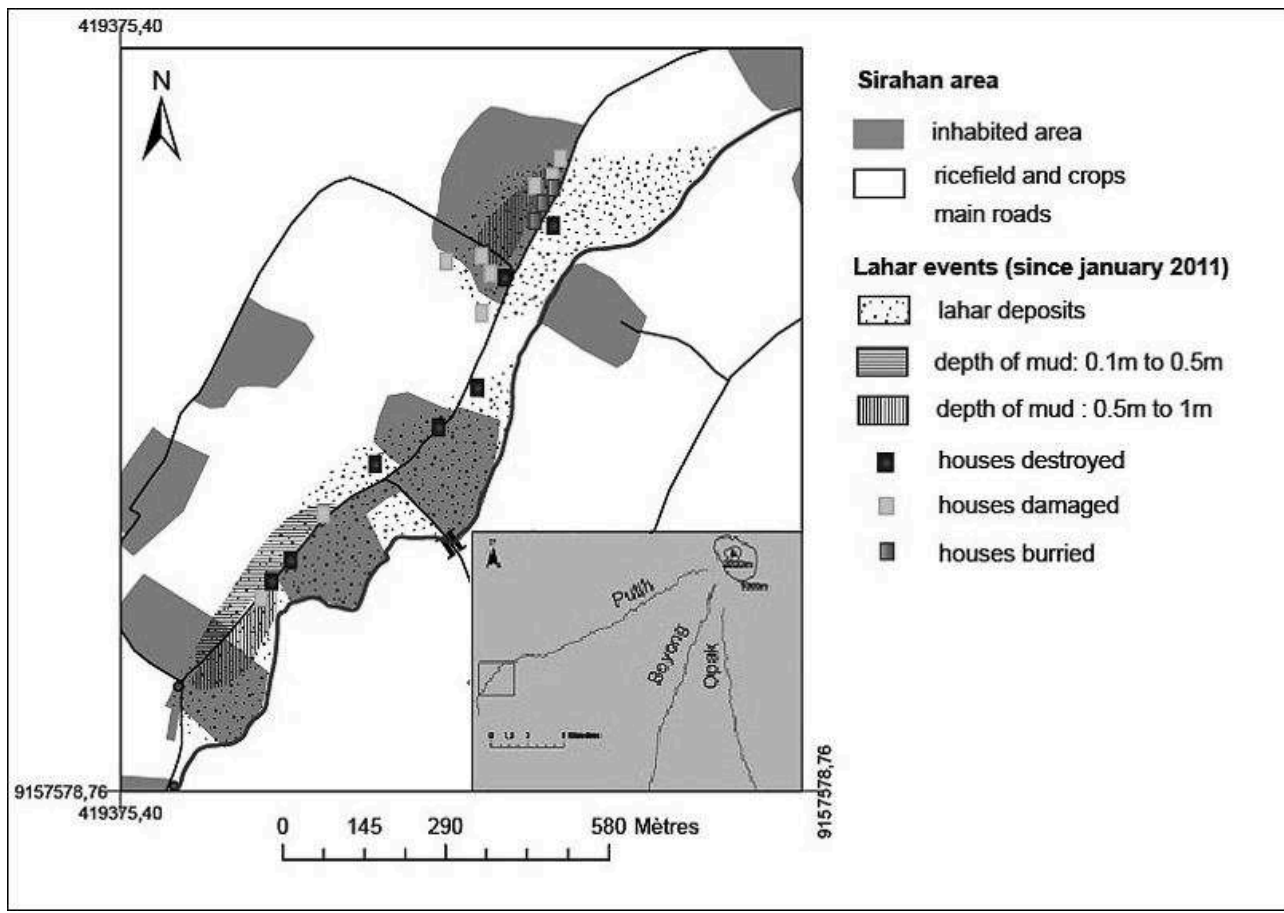

Source : De Belizal E., Lavigne F., Robin A.K. , 2011. Lahar disasters following the 2010 eruption of Merapi volcano (Indonesia). Regional Geography Conference UGI-IGU, Santiago, Chili,

14-19 novembre 2011

5 L'intérêt de ce sujet m'a poussée à poursuivre mes études en géographie physique, en master 1 et 2 de "dynamique des milieux et risques" (Université Paris 1), participant alors à l'étude qui a mis en relation des traces d'éruption connues à l'échelle mondiale et le volcan Samalas, situé sur l'île de Lombok ${ }^{2}$. En master 2, j'ai commencé à travailler sur les obsidiennes en Arménie, ce qui m'a permis d'obtenir ensuite une allocation doctorale. Ainsi, je me suis tournée vers la géomatique à mi-parcours de mes études en environnement, au départ comme un moyen d'obtenir un emploi plus appliqué dans le domaine des risques et, finalement, comme un outil au service de mes recherches. Outre l'apprentissage des SIG, ce qui m'a beaucoup aidé, c'est l'apprentissage de ce qu'est une carte avec ses règles de lecture et de construction. En environnement, l'approche globale et très ouverte des enseignements m'a donné une culture générale, tant au plan physique que social, mais aussi un esprit critique et davantage de recul, ce qui a certainement facilité mon intégration à une équipe de recherche interdisciplinaire.

6 - KL. À l'issue d'un BAC STL Physique, j'ai suivi une première année de BTS en mesures physiques, avant de bifurquer vers une licence de géographie à l'Université de Strasbourg, dont la formation me paraissait plus concrète. Après une L3 en environnement à l'Université Paris 7, j'ai souhaité compléter mes acquis en géomatique, choisissant alors une voie plus directement professionnalisante, celle de la licence professionnelle "environnement et géomatique". Cette formation a complété mes connaissances en environnement et m'a apporté des compétences techniques en géomatique. C'était un bon moyen de trouver un équilibre entre théorie et pratique.

7 - MLD. Après deux années de licence de géographie (spécialité Aménagement du territoire et cartographie), je me suis prise d'un engouement certain pour l'information 
géographique. Un stage librement effectué au Service Hydrographique et Océanographique de la Marine (SHOM) m'a permis de concrétiser professionnellement mon intérêt pour les cartes et l'environnement maritime. Déterminée à continuer dans cette voie, j'ai obtenu une licence professionnelle en Géomatique et environnement, qui m'a apporté une formation à la fois scientifique (par exemple en géomorphologie, en communication sur l'environnement, en aménagement) et une formation technique, dans le traitement des images spatiales et les SIG. Mon stage de licence a été effectué à l'Agence des Aires Marines Protégées (AAMP) de Brest, pour laquelle j'ai eu à constituer un atlas thématique des AMP françaises. L'Institut Français de Recherche et d'Exploitation de la Mer (IFREMER) m'a ensuite proposé un CDD d'une année afin de prendre en charge l'administration des données relatives aux dossiers d'extension du plateau continental français (EXTRAPLAC). Outre les fonctions administratives habituelles des SIG, j'ai pu appliquer mes connaissances sur le terrain en embarquant sur le navire l'Atalante pour une campagne océanographique en Polynésie. Cette mission (POLYPLAC) a pour but de démontrer une continuité géologique à l'Est du plateau marquisien afin d'étendre la ZEE française.

8 - FA. Je n'ai découvert la géomatique qu'en master 2, après avoir suivi des études de droit de l'environnement. J'envisageais de travailler dans l'aménagement du territoire ou la gestion de l'environnement, ce qui m'a poussée à élargir mon champ de compétences en intégrant une formation pluridisciplinaire me permettant notamment de me former à la conduite de projet et à la géomatique. J'ai pratiqué celle-ci lors de mon stage. Souhaitant acquérir une double compétence juridique et technique, j'ai ensuite intégré l'École des Eaux et Forêts pour suivre un mastère spécialisé en gestion forestière, au sein duquel j'ai pu approfondir ma pratique de la géomatique.

\section{Une utilisation complète et diversifiée des outils de la géomatique}

9 De l'acquisition des données à l'édition cartographique, du GPS au logiciel de DAO en passant par le SIG, la palette des outils de la géomatique utilisés est complète et diversifiée, dans les différents métiers exercés par nos cinq interviewés.

10 - EL. J'utilise principalement la géomatique sur des missions en évaluation de risques, où les outils associés à la discipline permettent d'une part de fournir des analyses spatiales et/ou des simulations puissantes à un instant $\mathrm{T}$, ou sur des périodes plus larges en $\mathrm{T}+\mathrm{x}$, et d'autre part de constituer des supports de communication parlants pour nos collaborateurs et/ou clients par la production de visuels spécifiques (cartes ou vidéos). Bien souvent les données que je manipule proviennent des organismes tels que l'IGN ou le BRGM, mais parfois je suis amené à créer de la donnée à partir de vues aériennes ou par des relevés de terrain.

11 - AKR. Ce sont essentiellement les SIG que j'utilise, à la fois en laboratoire et sur le terrain (avec le SIG-nomade dans ce cas), sur un rythme quasi-quotidien. Les SIG me permettent au départ de préparer mes missions de terrain et, in fine, de constituer une cartographie de synthèse ou d'illustrer mes travaux scientifiques. Le SIG-nomade m'offre la possibilité d'annoter et de compléter in situ mes observations géoréférencées. 
12 - KL. À partir des données d'études issues des différents services, fichiers du nouveau tracé à grande échelle, données environnementales et autres, je réalise l'implantation de la ligne ferroviaire dans son environnement géographique avec le SIG (ArcGIS) pour pouvoir le restituer à différentes échelles. Le service doit pouvoir à terme constituer une base commune pour l'ensemble des lignes ferroviaires et mettre à disposition ces informations. Ainsi autant que possible, j'intègre les données mises à disposition par les grands organismes tels que l'IGN, le BRGM et les ministères. Il y a donc un gros travail de récupération de données, puis de mise en référence commune et enfin de cartographie pour répondre aux besoins de communication des services. La demande peut se faire en cartes finalisées ou en données organisées. Dans ce cas, il est nécessaire de produire aussi les métadonnées.

13 - FA. À l'échelle intercommunale, la géomatique permet d'acquérir des données sur le territoire, d'en améliorer la connaissance et d'en faciliter la gestion. Le service SIG et les agents créent des bases de données spatialisées pour gérer les différentes politiques (cadastre, zonages PLU...) ou les comptages routiers. Ces données servent à la mise en place d'un SIG intercommunal et permettent de mieux gérer le territoire. L'intérêt est de mutualiser les données avec les communes et de les diffuser au-delà (partage de certaines données sur le site GéoBretagne par exemple). Les différents services des communes et de la communauté de communes ont accès à des applications métiers (SIG en ligne) mises en place par la communauté de communes. Le SIG contribue également à la gestion de certaines politiques publiques (entretien et suivi des réseaux d'assainissement par exemple) et à la communication (le service SIG a créé des applications en ligne sur la mobilité, les sentiers de randonnées). Je dirais que mon utilisation des outils SIG reste assez classique, allant de l'acquisition de données et la gestion de bases de données à des analyses croisées de données et de la cartographie.

14 - MLD. Le premier travail qui m'est confié consiste en l'acquisition de données. Notre principale source d'informations est l'Institut Géographique National. Différentes commandes sont passées selon les besoins du bureau. Il s'agit, le plus souvent, de fonds de plans (cartes numériques) à l'échelle $25000^{\mathrm{e}}$ ou $100000^{\mathrm{e}}$ qui nous servent de supports de travail. Parfois nous devons construire nos propres données géographiques. C'est par exemple le cas après une observation terrain ou d'après certains ouvrages. Il est ensuite important de veiller à la gestion de toutes ces informations : les classer de manière logique dans des répertoires de données, puis les mettre à jour, en modifiant certaines informations des tables attributaires et des métadonnées. La combinaison des différentes sources d'information nous permet à terme de trouver les meilleurs emplacements pour la construction de parcs éoliens. Enfin, des cartes sont réalisées afin de mettre en évidence les différentes analyses effectuées. C'est une étape relativement importante puisque c'est souvent ce que l'on retiendra d'un rapport d'étude et ce qui servira de support lors des réunions. 
Illustration 2 - Diagnostic paysager pour l'implantation de zones de développement éolien

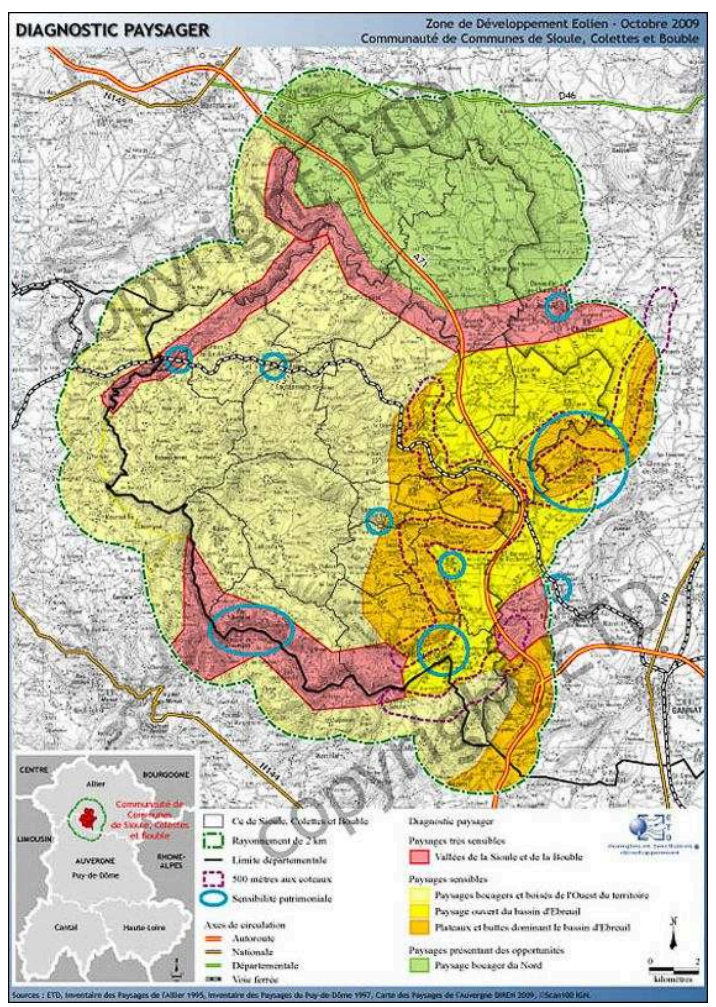

Combinaison d'informations permettant de déterminer les meilleures implantations possibles pour des éoliennes au regard de critères paysagers.

Sources: ETD, laboratoire des paysages de l'Allier 1995 ; inventaire des paysages du Puy-de -Dôme 1997 ; carte des paysage de l'Auvergne 2009.

15 - AKR. Dans le domaine géologique, les SIG me permettent aussi de constituer des "minutes de terrain", c'est-à-dire de donner les limites des affleurements ou des unités géologiques visibles sur le terrain (coulées de lave, affleurements d'obsidiennes).

16 - FL. Dans le cadre de mes projets, je suis amenée à acquérir des données et créer des bases de données plus spécifiques (relevés GPS sur les panneaux d'affichage publicitaire, comptages routiers...), qui servent ensuite du diagnostic au suivi des actions. Je me sers de ces outils pour réaliser des études, des diagnostics préalables. Ils ont l'intérêt de faciliter la prise de décision politique et technique. Par exemple, je travaille sur le schéma directeur des déplacements. Ce document consiste à programmer les différentes infrastructures à mettre en place (aires de covoiturage, pistes cyclables..). J'utilise les SIG pour décider où faire passer les liaisons cyclables, quels types d'aménagements mettre en place. Ils me permettent de croiser des données, superposer des couches (trafic, distance, pente), évaluer différents scénarios. Avec la géomatique, on peut facilement faire parler le territoire. Pour l'affichage, ils m'ont permis d'analyser les priorités de gestion et le type de gestion à mettre en place en différents secteurs. Les outils de la géomatique me permettent également de suivre et gérer les actions : par rapport aux données d'état des lieux, on peut facilement mettre à jour les bases de données et réaliser des bilans. Grâce à eux, l'évaluation des politiques publiques peut devenir plus systématique (évolution de l'affiche publicitaire illégal sur le territoire, nombre de kilomètres de pistes cyclables créés par rapport aux objectifs initiaux etc.). 
17 - KL. Pour traiter les mises en pages, il est parfois nécessaire de faire appel à des outils de DAO. J'ai aussi la possibilité de participer à la définition de la charte graphique des productions.

18 - ML. J'utilise moi aussi des outils des DAO pour un meilleur rendu des travaux cartographiques. Une charte graphique est respectée afin que l'ossature des cartes soit cohérente pour toutes les illustrations figurant dans le rapport d'étude. Ces outils sont également utilisés lors de la réalisation de posters d'information. Ainsi, mon travail est centré sur les SIG de façon globale, de l'acquisition de données jusqu'à la cartographie.

19 - FA. De mon point de vue, les cartes sont aussi très utiles pour communiquer auprès des élus et du grand public (application cartographique en ligne sur la mobilité par exemple).

\section{La géomatique, un atout dans la gestion de territoires difficiles à appréhender}

20 La géomatique est essentielle dans chacun des métiers de l'environnement évoqués dans ces interviews. Elle est l'atout indispensable pour une gestion optimisée et centralisée d'informations multiples, sur des territoires difficiles à appréhender.

21 - EL. Dans mes activités, les outils de la géomatique sont un réel atout, puisque je travaille principalement sur des projets de grande envergure (par exemple, une évaluation du risque inondation sur des milliers de sites ou du risque érosion sur des réseaux de plusieurs milliers de kilomètres). Même si la collecte d'informations peut être très longue, les SIG me permettent au final un gain de temps non négligeable mais je ne pense pas que ces outils soient absolument indispensables. Certes, pour moi ils sont intéressants à exploiter et me permettent des gains en termes de temps, de précision, de communication, parce que je suis capable de les exploiter. Mais certains collaborateurs novices ou étrangers à la discipline réalisent très bien ce genre de missions sans la géomatique.

22 - AKR. Les outils de la géomatique sont indispensables pour comprendre comment s'organise un terrain à différentes échelles. Le SIG permet de produire ce résultat sans perdre trop d'informations lors du changement d'échelle. Par exemple, dans ma thèse, le contexte géologique doit être compris à la fois à l'échelle locale (recherche des affleurements d'obsidienne) mais également à l'échelle régionale, du fait des distances entre chacun des volcans étudiés et des effets globaux de la tectonique. Ainsi, un affleurement actuel aurait pu être récemment "mis à nu" par une évolution tectonique, alors qu'il n'était pas accessible à l'époque où l'obsidienne était exploitée. La diffusion des obsidiennes a couvert une région plus grande encore, allant au Néolithique de l'Égypte à la Géorgie : le SIG facilite ainsi la recherche de chemins de diffusion, à travers l'utilisation de la topographie et de l'hydrographie.

23 - KL. Les outils de la géomatique sont primordiaux dans le sens où ils interviennent pour l'assemblage, la visualisation et l'interrogation des bases. De même que pour la mise en partage via des serveurs ou des conversions en différents formats de fichiers utilisés. Ils assurent la mise en conformité des informations et permettent d'en garantir le descriptif et la qualité.

24 - FA. À mon sens, nous avons à gérer de plus en plus de données. Les bases de données classiques ont leurs limites dans l'aménagement du territoire et la gestion de 
l'environnement. Les territoires peuvent être très différents d'un point à un autre. Pouvoir spatialiser les données permet une planification plus optimale et une gestion plus efficace du territoire. Je pourrais difficilement m'en passer ; elles nous permettent justement de réfléchir aux choix politiques à l'échelle du territoire, voire au-delà, et d'éviter de faire des choix simplement opportunistes.

Illustration 3 - Extrait du dignostic de l'affichage publicitaire sur la communauté de communes Côte d'Émeraude

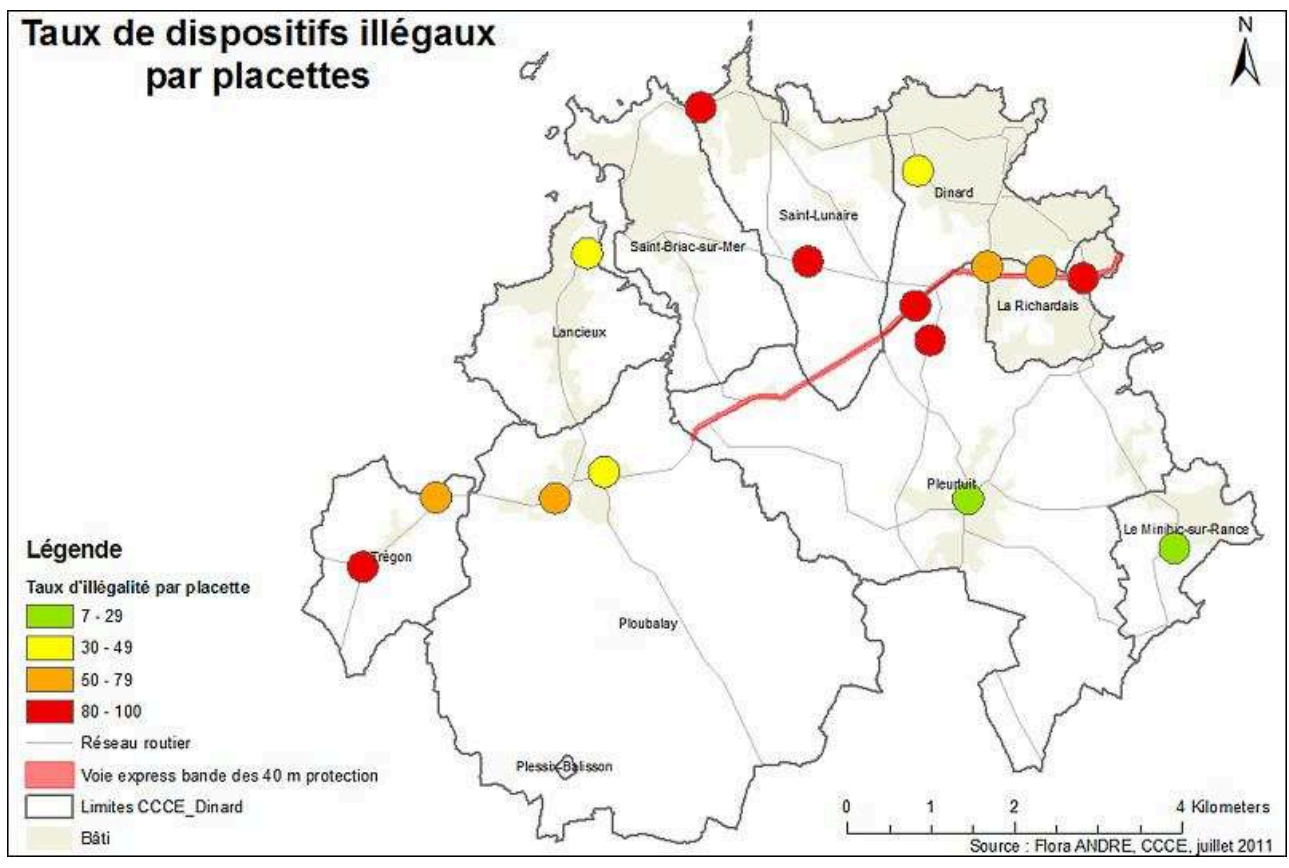

Source : F. André, CCCE, 2011.

\section{Géomatique et environnement : de nouveaux métiers ou faire-valoir d'un CV?}

À la question de savoir si l'intégration de la géomatique dans l'environnement conduit vers de nouveaux métiers ou si elle n'est qu'un simple faire-valoir sur leur $\mathrm{CV}$, les interviewés répondent que la double compétence géomatique et environnement est un réel plus pour la compréhension des enjeux et leur traduction en informations, utiles à la prise de décision. C'est aussi une valeur ajoutée reconnue en tant que critère d'embauche.

- FA. De mon point de vue, la géomatique est une compétence que je mets en avant dans mon CV. Je pense que les outils vont évoluer et que les savoirs faire des agents territoriaux travaillant sur l'aménagement du territoire vont évoluer en ce sens.

27 - E.L. Personnellement, de simple faire-valoir au départ, je considère désormais la géomatique comme une réelle compétence dans mon CV. Mais ce n'est pas forcément le cas pour tous, puisque cette discipline reste peu (re)connue encore. Mais il ne faut pas hésiter à mettre en avant ces outils et démontrer leur utilité dans la structure où l'on évolue. 
28 - AKR. La géomatique apporte un regard plus critique sur la construction des cartes et sur la diffusion de l'information, de façon générale comme dans le domaine particulier de l'environnement. Pour moi, c'est un support dans l'aide à la synthèse mais aussi dans l'aide à la décision.

29 - KL. La géomatique pourrait se présenter comme un domaine à part entière à large spectre. Néanmoins, pour qu'elle prenne sens, elle doit s'associer aux autres domaines, elle se trouve au carrefour des métiers. Avoir la double compétence géomatique et environnement est un réel plus car cela aide à la compréhension des sujets et à une définition correcte des objectifs.

\section{Relations environnement et géomatique, quel regard?}

Quel est le regard de nos interviewés, même critique, sur les relations entre environnement et géomatique ? A priori, on serait arrivé à un accord presque parfait, mais il demeure quelques fausses notes au plan de la reconnaissance institutionnelle.

31 - AKR. De mon point de vue, la géomatique sert assez bien les questions d'environnement, mais on l'utilise surtout dans le domaine de la géographie physique, donc davantage pour des paramètres physiques que pour des paramètres humains. Par exemple dans le domaine des risques, les aléas me semblent plus souvent cartographiés que la vulnérabilité ou les dommages.

32 - KL. Oui mais pas seulement en physique car les besoins de de la géomatique et de l'environnement se complètent aussi pour les aspects d'aménagement du territoire comme les réseaux. L'environnement va apporter sa thématique et ses informations écrites, tandis que la géomatique va les restituer dans un contexte donné.

33 - MLD. Pour moi, la place de la géomatique dans mon bureau d'étude est évidente. En effet, c'est un outil très pratique pour analyser des informations. Par exemple, en croisant la couche de données sur la zone où les éoliennes sont susceptibles d'être implantées avec la couche sur les voies empruntées par les oiseaux migrateurs, on évite de perturber l'écosystème. Les SIG permettent, également, de faire le lien entre les ingénieurs, les décideurs, et le grand public en "vulgarisant » les informations brutes en supports plus pédagogiques que sont les cartes. La géomatique s'accorde, pour moi, complètement à l'environnement, et n'a pas fini de se développer dans le domaine des énergies renouvelables.

34 - EL. Évidemment les questions environnementales soulèvent beaucoup de paramètres parfois différents - à prendre en compte et la géomatique permet cet exercice. Le plus contraignant est souvent de disposer des données adéquates. Pour ma part, je rencontre quotidiennement des barrières sur les plans de l'économie, de l'existence et de la fiabilité. Tout d'abord, les données sont très coûteuses; ensuite, elles ne sont pas toujours disponibles et il est bien souvent nécessaire de les (re)produire ou de les (re)travailler; enfin, on observe des décalages d'une source à une autre pour une même donnée.

- AKR. C'est pour cela qu'il faudrait, à l'avenir, plus d'interactivité entre acteurs autour de la construction d'un SIG: entre scientifiques de différentes disciplines, entre scientifiques et acteurs ou gestionnaires locaux du risque, de façon à constituer des bases de données communes et surtout des cartes communes, construites collectivement à partir des informations tirées du SIG. Car le "SIG visuel" aidera de 
beaucoup à la prise de décision, à la verbalisation des problématiques, à la participation sociale, à une meilleure prévention dans le champ des risques...

- KL. L'environnement et la géomatique ont une relation essentielle ! C'est une nouvelle façon de " consommer " l'information, avec un accès et un partage beaucoup plus rapides.

37 - EL. La géomatique est certes pratique et puissante avec des applications quasi-infinies - je note aussi un intérêt croissant de nos clients pour les outils SIG - mais dans un cadre professionnel les barrières citées peuvent forcer à envisager d'autres solutions.

- FA. Aujourd'hui les outils géomatique servent la gestion de l'environnement et l'aménagement du territoire pour les différentes raisons évoquées (efficacité, meilleure connaissance du territoire, aide à la décision, suivi...). Cependant, un gros travail reste à effectuer pour mutualiser les données, développer le partage de données opensource (GéoBretagne par exemple). Le fait que chaque échelon territorial dispose de données mais ne les communique pas constitue une vraie difficulté. Le problème se pose ainsi pour la thématique transport. Les réseaux de transports collectifs appartiennent à différents opérateurs (communes, communauté de communes, communauté d'agglo, Conseil général, Rennes Métropole...) et peu de données sont partagées. Or les déplacements des personnes ne se limitent pas aux frontières administratives.

Par ailleurs, les SIG deviennent de plus en plus essentiels pour les territoires, mais ces compétences techniques ne sont pas reconnues par les statuts de la fonction publique territoriale. En effet, la plupart des agents qui utilisent les outils SIG dans notre collectivité sont issus de formations universitaires. Les agents en charge de la cellule SIG eux-mêmes ne peuvent pas prétendre au concours d'ingénieur territorial en externe, réservé aux ingénieurs. Nous passons donc les concours de la filière administrative alors que nos missions sont techniques. Après 5 ans d'expérience, nous avons enfin accès au concours d'ingénieur en interne, mais les épreuves sont beaucoup plus éloignées de nos formations d'origine (mathématiques etc.).

\section{NOTES}

1. Coulées de débris d'origine volcanique.

2. Lavigne F., Degeai J.-P., Komorowski J.-C., Guillet S., Robert V., Lahitte P., Oppenheimer C., Stoffel M., Vidal C. M., Surono, Indyo Pratomo, Wassmer P., Irka Hajdas, Danang Sri Hadmoko, and de Belizal E., September 30, 2013. Source of the great A.D. 1257 mystery eruption unveiled, Samalas volcano, Rinjani Volcanic Complex, Indonesia. PNAS, October 15, 2013, vol. 110, no. 42, p. 16742-16747. doi : 10.1073/pnas.1307520110. 


\section{AUTEURS}

\section{CÉCILE HUET}

Cécile Huet, cecile.huet@ensg.ign.fr, est Ingénieur des travaux géographiques et cartographiques de l'État, enseignante au département de cartographie et d'analyse de l'information géographique, ENSG et co-responsable (pour l'ENSG) du M2 DDMEG.

\section{NICOLAS PAYET}

Nicolas Payet, nicolas.payet@ensg.eu, est enseignant au département de cartographie et d'analyse de l'information géographique, ENSG et co-responsable de la Licence Pro Géomatique et Environnement.

\section{LYDIE GOELDNER-GIANELLA}

Lydie Goeldner-Gianella, géographe, lydie.goeldner-gianella@univ-paris1.fr, est Professeur des universités à l'Université Paris 1 Panthéon-Sorbonne, UMR 8586 PRODIG et co-responsable (pour la géographie) du M2 DDMEG. 